\title{
Large bowel obstruction due to an impacted barolith - a delayed complication of a barium study
}

\author{
M.P. Saunders \\ Department of Surgery, Greenwich District Hospital, Greenwich, London SE10 9HE, UK.
}

Summary: Complications of barium sulphate studies abound in the literature but there are very few recorded instances of large bowel obstruction due to inspissated barium and faeces forming a 'barolith'. A patient who suffered this complication is reported.

\section{Introduction}

Valladares' coined the term 'bariolite' or 'barolith' for inspissated barium sulphate associated with faeces. His original paper in 1950 describes 10 cases of obstructive problems secondary to the diagnostic use of barium sulphate. In 8 of the cases, divided equally between small bowel studies and barium enemas, no underlying colonic pathology was identified. Cases of appendicitis following barium meal studies are reported, ${ }^{2}$ the lumen of the appendix being obstructed by barium. A more serious and potentially lethal complication due to inspissated barium is recorded in a case report ${ }^{3}$ of perforation of the sigmoid colon within 20 hours of a barium meal in the absence of colonic pathology.

This case report describes a 58 year old man who presented with large bowel obstruction due to an impacted 'barolith' complicating a barium meal examination performed more than a year earlier. Factors contributing to barolith formation are discussed and actions to avoid such a repetition are recommended.

\section{Case report}

A 58 year old man was admitted as an emergency complaining of several days of colicky lower abdominal pain, abdominal distension and vomiting. He claimed to have passed flatus but had not defaecated for at least one week. There was no history of haematemesis, melaena or significant urinary symptoms. He suffered chronic ill health including diabetes mellitus, ischaemic heart disease, epilepsy, peripheral vascular disease and depression, each currently requiring medication

Correspondence: M.P. Saunders F.R.C.S., Surgical Unit, Guy's Hospital, London SE1 9RT, UK.

Accepted: 13 March 1990 including opiate analgesia and tricyclic antidepressants. He had poor mental function and was a reformed alcoholic. His past surgical history was equally extensive; 20 years of chronic duodenal ulcer symptoms were initially treated by gastroenterostomy (1968) only to be complemented by vagotomy and pyloroplasty (1972) and further entero-enterostomy (1972) and modified to a Polya gastrectomy (1974) for a refractory stomach ulcer. A gastrinoma has never been identified. In 1986 he had a cholecystectomy. During this protracted period of ulcer diathesis he had repeated gastroscopies though in 1987 a barium meal was requested. With the abeyance of his dyspeptic symptoms he was lost to follow-up from this date to the present admission. To the best of our knowledge no further barium studies had been performed.

On examination he appeared dishevelled and was confused. He was not clinically jaundiced, anaemic nor dehydrated and was apyrexial and normotensive with stable cardiovascular and respiratory function. There were multiple surgical scars on his abdomen. Tenderness was confined to the left upper quadrant of the abdomen and no masses were palpable. Bowel sounds were obstructive in nature. Rectal examination revealed impacted faeces.

Initial investigations noted a raised white cell count predominantly leucocytic, normal haemoglobin, renal function and serum amylase. Abdominal films indicated partial large bowel obstruction apparently due to a large opacity $(>6 \mathrm{~cm}$ diameter) at the splenic flexure, gas was present in the left colon and rectum. A regimen of intravenous fluids and nasogastric aspiration was commenced and a disposable enema given; initially there was much improvement and some bowel action.

Several days later, however, his condition 


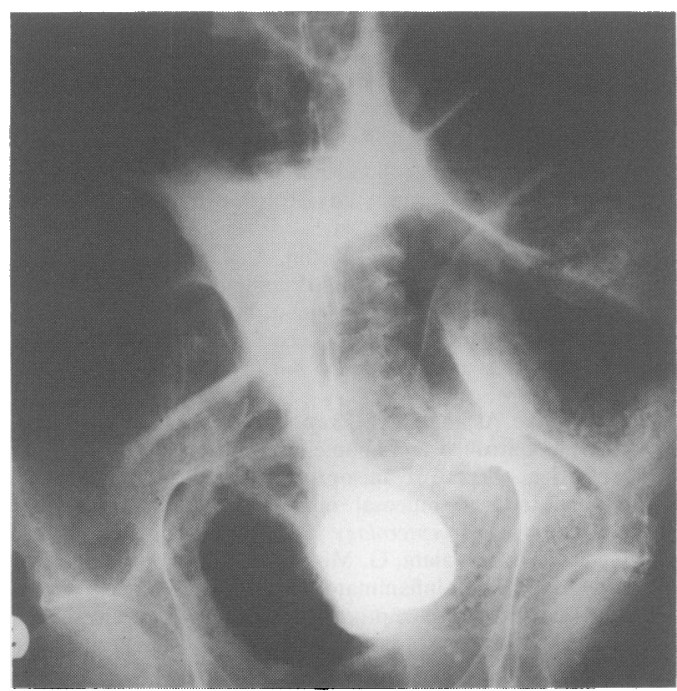

Figure 1 Anterio-posterior plain abdominal X-rays in supine position.

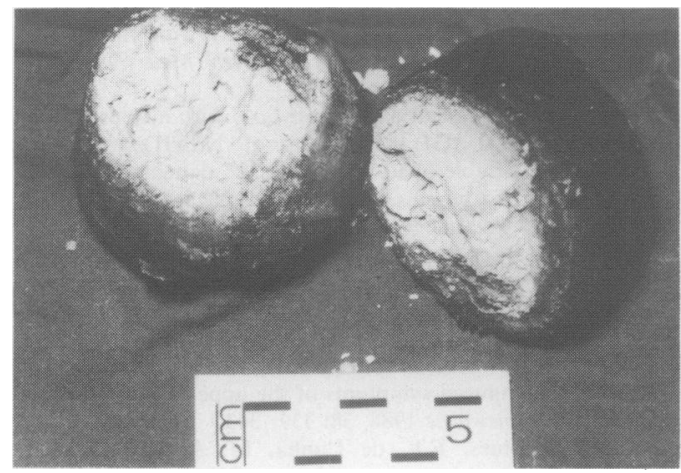

Figure 2 Removed barolith, divided, to reveal inspissated barium sulphate.

deteriorated with signs of complete intestinal obstruction and impending perforation. Abdominal $\mathrm{X}$-rays confirmed large bowel dilatation and the opacity had shifted to the distal sigmoid colon (Figure 1). The mass was palpable per rectum but not visible via sigmoidoscopy because a sleeve of intussuscepted mucosa intervened. Despite insufflation of the rectum and indirect contact pushing with the sigmoidoscope the mass remained

References

1. Valladares, C.D.P. Bariolitos, Rev Brasil Gastroenterol 1950 , 2: 595-608.

2. Gubler, J.A. \& Kukral, A.J. Barium appendicitis. J Int Coll Surgeons 1954, 21: 379-384.

3. Serjaent, J.C.B. \& Raymond, J.A. Perforation of apparently normal colon after a barium meal. Lancet 1952, ii: 1245-1246. immoveable. Through an oblique muscle dividing incision in the left iliac fossa a double-barrelled colostomy was fashioned, the barolith (Figure 2) milked back and removed. The patient recovered and plans are to close the stoma in the near future.

\section{Discussion}

In a review of complications of barium sulphate studies $^{4}$ a rare case of an asymptomatic adherent non-obstructive barolith of the caecum present 2 years after previous studies is described. In such instances surgical removal is recommended because of the barolith's adherence and resistance to purging as well as the possibility of intussusception, obstruction, ulceration and perforation of the colon.

Factors contributing to a barolith formation include abnormal anatomy, gut immotility or underlying pathology ${ }^{4}$ and the patients' general health, age, ${ }^{5}$ immobility, state of hydration ${ }^{3}$ and diet. In the presence of such risk factors a follow-up film several days later is recommended and, if barium residue remains, laxative treatment should be administered. The routine use of laxative treatment after barium meal studies in elderly patients is advised $^{5}$ to reduce the incidence of colonic retention of the barium sulphate.

We suggest that the barolith described in our patient initially formed within the stomach following the barium meal in 1987. Due to incomplete gastric emptying, the retained barium became inspissated and passed into the jejunum via the gastroenterostomy and subsequently migrated into the large bowel where it increased in size with adherent faecal matter to form an obstructing mass.

The patient's poor condition, irregular diet and large opiate plus anticholinergic drug intake undoubtedly resulted in the delayed transit of barium and enabled the barolith to form.

\section{Acknowledgement}

I would like to thank Mr T.A. Harrison F.R.C.S., Consultant Surgeon, Greenwich District Hospital, for his help in the preparation of this manuscript and permitting me to report this case.
4. Dixon, G.D., Ferris, D.O. \& Hodgson, J.R. Unusual complication of barium studies. Report of a case of adherent caecal barolith. AJR 1967, 99: 106-111.

5. Prout, B.J., Datta, S.B. \& Wilson, T.S. Colonic retention of barium in the elderly after barium-meal examination and its treatment with lactulose. Br Med J 1972, 4: 530-533. 